\title{
Resource preferences and the emergence of individual niche specialization within populations
}

\author{
Daniel Sol${ }^{1}$, Oriol Lapiedra ${ }^{1}$, César González-Lagos ${ }^{2}$, and Miquel De Caceres ${ }^{3}$ \\ ${ }^{1}$ CREAF \\ ${ }^{2}$ Universidad Bernardo O'Higgins \\ ${ }^{3} \mathrm{CTFC}$
}

November 19, 2020

\begin{abstract}
Growing evidence that individuals of many generalist animals behave as resource specialists has attracted research interest for its ecological and evolutionary implications. Although variation in resource preferences is critical for developing a general theory of individual specialization, it remains to be shown whether diverging preferences can arise among individuals sharing a similar environment and whether these are stable enough to be ecologically relevant. We addressed these issues by means of common garden experiments in feral pigeons (Columba livia), a species known to exhibit resource specialization in the wild. Food-choice experiments on wild-caught pigeons and their captive-bred descendants showed that variation in food preferences can easily arise within a population and that this variation may represent a substantial fraction of the population niche. However, a cross-fostering experiment revealed that the genetic and early common-environment components of food preferences were low, reducing their stability and eroding niche variation in the long-term.
\end{abstract}

Resource preferences and the emergence of individual niche specialization within populations Daniel Sol ${ }^{1,2^{*}}$, Oriol Lapiedra ${ }^{1 *}$, Cesar González-Lagos ${ }^{3,4^{*}} \&$ Miquel de Cáceres ${ }^{1,5}$

${ }^{1}$ CREAF, Centre for Ecological Research and Applied Forestries, Cerdanyola del Vallès, Catalonia 08193, Spain

${ }^{2}$ CSIC, Spanish National Research Council, CREAF-UAB, Cerdanyola del Vallès, Catalonia 08193, Spain

${ }^{3}$ Centro de Investigación en Recursos Naturales y Sustentabilidad (CIRENYS), Universidad Bernardo O'Higgins, Santiago, Chile

${ }^{4}$ Centre of Applied Ecology and Sustainability (CAPES)

${ }^{5}$ Joint Research Unit CTFC-AGROTECNIO, E25280 Solsona, Catalonia, Spain

${ }^{*}$ Shared first-author

Number of words in the abstract (149)

Number of words in the manuscript as a whole, excluding abstract, references, and captions (4987)

Number of references in main text (45) Number of figures (5) and tables (1) in main text

Name and complete mailing address of the person to whom correspondence should be sent: Daniel Sol, CREAF-CSIC, Cerdanyola del Vallès, Catalonia E-08193, Spain. TEL: +34 93-5814678. E-MAIL: d.sol@creaf.uab.cat 
Author contributions: DS, OL and CGL conceived and designed the study; OL and CGL collected data; DS, OL and MD conducted the analyses; MD developed a new framework to measure niche breadth; DS wrote the manuscript and all authors edited and approved it.

Data accessibility statement: We confirm that, should the manuscript be accepted, the data supporting the results will be archived Dryad, and the data DOI will be included at the end of the article.

Abstract. Growing evidence that individuals of many generalist animals behave as resource specialists has attracted research interest for its ecological and evolutionary implications. Although variation in resource preferences is critical for developing a general theory of individual specialization, it remains to be shown whether diverging preferences can arise among individuals sharing a similar environment and whether these are stable enough to be ecologically relevant. We addressed these issues by means of common garden experiments in feral pigeons (Columba livia), a species known to exhibit resource specialization in the wild. Food-choice experiments on wild-caught pigeons and their captive-bred descendants showed that variation in food preferences can easily arise within a population and that this variation may represent a substantial fraction of the population niche. However, a cross-fostering experiment revealed that the genetic and early common-environment components of food preferences were low, reducing their stability and eroding niche variation in the long-term.

Keyword: Niche variation; individual differences; decision-making; learning the niche; behavioural plasticity; heritability of behaviour; cross-fostering experiment.

Niche variation - in which individuals consistently use a subset of the resources available for the population (Van Valen 1965) - is a widespread phenomenon in vertebrate and invertebrate taxa (Bolnicket al. 2003). Although much of this variation can be explained by either ontogenetic niche shifts or sexual dimorphism (Schoener 1986; Price 1987), resource-use variation is observed even among individuals of the same age and sex (Araújo et al. 2011). In Brünnich's guillemots (Uria lomvia), for example, individuals show foraging specializations in diving technique, which are then reflected in differences in the type of fish and marine invertebrates they mostly capture (Woo et al. 2008). This type of niche variation - known as individual specialization - has attracted considerable interest for its ecological and evolutionary implications (reviewed in Bolnicket al. , 2003, 2011; Dall et al. , 2012). Thus, individual specialization has been related to a wide array of ecologically and evolutionary relevant processes, including skill-pools, population stability, species coexistence, extinction, niche evolution and speciation (Giraldeau 1984; Sol et al. 2005; Tinker et al.2008; Bolnick et al. 2011). Surprisingly, however, how individual variation in resource use is generated and maintained within populations is still insufficiently understood (Araújo et al. 2011; Bolnicket al. 2011). As a consequence, the actual relevance of individual resource specialization in ecology and evolution remains unclear.

The existence of variation among individuals in resource preferences is central for developing a general theory of individual specialization. As pointed by Bolnick et al. (2003), if a population is found to be composed of individuals that consistently prefer different subsets of available foods, few would object to considering such individuals specialists. However, why should individuals sharing a same environment end up using only a subset of all available resources? Classic optimal foraging theory predicts that organisms' food choices should maximize some currency linked to fitness, like energy intake (Pyke 1984; Shettleworth 1985). It follows that if individuals behave optimally, they should converge in the way they use the resources rather than diverge.

Theoretical models suggest, however, that specialization may still occur if preferences are state-dependent, that is, if the decisions of individuals are modulated by their morphological, physiological and psychological features (Houston \& McNamara 1999). Araújo et al. , (2011) discussed two main ways how this can occur. One is when phenotypic variation produces among-individual differences in the ability to detect, capture, handle, or digest alternative prey. The second is when individuals use different optimization criteria in resourcechoice, for example because they have different energetic requirements (Schoener 1971). Both mechanisms may not only cause individuals to prefer some resources over others, but they may make preferences to be 
transmitted to offspring if the state-traits that have driven them are themselves heritable. Thus, although preferences can be extremely plastic, they can change more slowly if optimal choices are connected to a slow-changing state variable (Luttbeg \& Sih 2010).

Resource specialization may arise in yet another way, that is, through learning (Tinker et al. 2009). Although behaviour, physiology and morphology may set the limits to what an individual can eat, food preferences within these boundaries may arise through positive feedbacks (sensu Dingemanse \& Wolf 2010), where early experiences in the use of certain foods reinforce (if positive) initial choices (Dridi \& Lehmann 2015). A variety of situations may make individuals to have distinct early food experiences, including different perception of risk or spatially and/or temporally variation in resource availability. There is indeed growing evidence that learning is crucial for animals to develop their own niche (Slagsvold \& Wiebe 2007; Tinker et al. 2009). Given that resource preferences will be environmentally induced rather than constrained by the genetic architecture, however, it is not obvious how learned preferences will be maintained within a population. One would expect that as individuals are exposed to positive food experiences, their preferences will continue to expand rather than remain narrow. Yet, learning may still cause stable resource preferences if adopting new foods is cognitively costly, for example because it requires to learn a new foraging technique (Partridge \& Green 1987; Tinker et al. 2009). Learned preferences may also be maintained if they are phase-sensitive, that is, if they occur at a young age and after that individuals acquire an aversion to explore and incorporate new foods (Greenberg \& Mettke-hofmann 2001; Slagsvold \& Wiebe 2007).

Despite the importance of resource preferences in constructing a general theory of individual specialization, there has been little effort to assess whether individuals within populations consistently vary in resource preferences and to what extent this variation remains stable over time (Araújo et al. 2011; Bolnick et al. 2011). One reason of this neglect is that resource preferences are difficult to quantify. A major confounding effect is resource availability (Moon \& Zeigler 1979; De Cáceres et al. 2011). Preference is the likelihood that an individual selects a given item when offered alternative choices on an equal basis (Johnson 1980). If a particular food is relatively scarce or is monopolized by a superior competitor, it may represent only a small proportion of the resources used even if the animal has a high preference for that food. Preference also implies that the likelihood of selecting a given item is higher than expected by chance. This means that resource preferences need to be measured in standardized replicated observations, which may also be challenging (Araújo et al. 2011). On the other hand, although one can measure individual differences in resource preferences, the challenge remains as to how interpret the differences. Given that individuals may use different optimization criteria to rank foods, interpreting preferences requires to consider the energetic and nutritional contents of food (Machovsky-Capuska et al. 2018). It also requires to consider the multidimensional nature of foraging behaviour, which includes the search, identification, handling, consumption and digestion of foods.

Perhaps the most tractable way to tackle the difficulties of assessing and interpreting food preferences is the use of common garden experiments. Despite concerns over the extent to which the mechanisms we observe in these experiments tap into those actually used by animals in the wild, food-choice experiments involving a variety of food types in exactly the same amount remain the best way to estimate individual preferences under different optimization criteria and reducing the complexities associated with the multi-dimensional nature of foraging behaviour (Pyke 1984). Combined with cross-fostering breeding experiments — where the effects of rearing environment and genetic influences may be disentangled (Slagsvold \& Wiebe 2007) — foodchoice experiments offer the opportunity to investigate 1) whether individuals exhibit consistent differences in resource preferences and 2) to what extent these differences have a genetic basis or are environmentallyinduced. Here, we address these questions in the Feral pigeon (Columba livia), a granivorous bird that has been instrumental in the study of decision-making theory (Inman et al.1987; Shettleworth 1987a) and that in the wild is known to exhibit individual differences in food use (Giraldeau \& Lefebvre 1985; Johnston \& Janiga 1995). Although previous experiments in pigeons have found evidence for a unitary preference for certain seeds (Moon \& Zeigler 1979; Inman et al. 1987; Shettleworth 1987a; Biedermann et al. 2012), some authors have suggested that individuals might also vary in their preferences (Moon \& Zeigler 1979; Giraldeau \& Lefebvre 1985). 
To investigate whether resource specialization reflect variation in food preferences, we conducted replicated food-choice assays in a common garden framework using pigeons from two wild populations. In addition, we conducted food-choice assays on their captive-bred descendants - half of them cross-fostered among neststo estimate the heritability, vertical transmission and reactions norms of the preferences by means of a quantitative genetics approach. Finally, we repeated the food-choice assays one year later to assess the longterm stability of food preferences. Following De Cacéres et al. (2011), the contribution of niche specialization to the total niche width of the populations was assessed based on a comprehensive framework that extends classic information theory by incorporating the energetic and nutritional resemblance between resources into the calculation of resource niche metrics. Characterizing the niche exclusively in terms of quantity and range of different resources would ignore that individuals may use different optimization criteria to rank foods. Moreover, it would bias estimation of niche breadth as the niche of individuals consuming the same number of foods will be considered equivalent even when some individuals are using more different types of foods (Colwell et al. 1971; De Cáceres et al. 2011).

\section{Methods}

We captured 42 adult feral pigeons (Columba livia) from two free-living populations located $50 \mathrm{~km}$ apart, one in Barcelona (DD coordinates: 41.38879, $-2.15899, \mathrm{n}=23$ pigeons) and another from Moià (41.81112-2.09839, $\mathrm{n}=19$ ). The two populations show substantial differences in their foraging ecology, with individuals from Barcelona spending more time foraging and relying more on active searching for food than on sit-and-wait strategies than those form Moià (Sol 2008).

After capture, individuals were measured (wing, tail, tarsus and beak lengths), banded with a unique combination of coloured rings, and randomly assigned to four outdoor aviaries of $2 \times 4 \times 2 \mathrm{~m}$ (8-12 individuals from the same population). Each aviary was provided with roosting sites, ad libitum food (a commercial mixture of food) and water. For the experiments, individuals were relocated in individual cages of 70x50x40 $\mathrm{cm}$ containing a feeder and a watering bowl. Experimental cages were located indoors, to homogenize the conditions in which assays were conducted. During assays, birds were observed from behind a blind to avoid disturbance by the observer. Six individuals were tested simultaneously, assigned at random within each of the four outdoor aviaries. Experiments were videotaped and behaviour was scored using the software Jwatcher (https://www.jwatcher.ucla.edu/). All animal care, husbandry, and experimental procedures were in accordance with the Spanish code of practice for the care and use of animals for scientific purposes and were approved by the Generalitat de Catalunya (0152S).

Food-choice experiment

To assess food preferences of individuals, we presented each pigeon with a six-section circular Plexiglas feeder containing $10 \mathrm{~g}$ of six different seed types (green peas, oats, popcorn maize, soybeans, sunflower seeds, and wheat), an amount large enough to avoid being depleted during the trial. The six food types were part of the diet of pigeons during the acclimation period, and they were chosen to provide variation in size and nutritional contents. Individuals were allowed to feed for 20 minutes. After this time, we removed the feeder and measured the amount of each food remaining with a digital precision balance. The amount of each seed type consumed per individual was estimated by subtracting this quantity from the initial 10g. Trials in which an individual did not eat were excluded from the analyses. To evaluate the consistency in the measure, each individual was tested in a battery of 4 trials conducted in two consecutive days. In the first daily trial, individuals were tested under food-deprived conditions whereas in the second they had already eaten. Thus, our experimental design allowed us to assess the extent to which fasting influenced food choice (Moon \& Zeigler 1979). For some individuals (12 from Moià, 14 from BCN and 28 raised in captivity), long-term consistency in food preferences was evaluated by repeating the food choice experiment a year later (two trials in one day).

Captive breeding and cross fostering experiment

We separated 16 breeding pairs and placed them into breeding cages with material to build the nest and ad libitum mixture of food. We used pairs that were spontaneously formed in the aviaries, but we removed 
eggs for a few weeks to ensure male paternity. Because pigeons lay almost invariably two eggs (Johnston \& Janiga 1995), we randomly cross-fostered one of the two chicks. Nestlings were cross-fostered when two days old, ensuring that age difference with their new brother was less than two days. Because we did not have a pedigree for all individuals, we matched each offspring with its biological parents and assumed that pairs were distantly related to each other.

\section{Analyses}

From the amount of food each individual consumed in the food preference tests we derived metrics aimed at estimating niche centre, breadth and overlap (De Cáceres et al. 2011). We computed a distance matrix between food types based on their nutritional content (hydrocarbons, fat and proteins) while also taking into account the physical characteristics of each food type (length and width). These variables, standardized in order to remove differences due to units of measurement, were used to calculate the Euclidean distance between pairs of seed types. The niche centre in this resource space was computed by means of a principal coordinate analysis (PCoA), averaging the coordinates of the resources preferred by the individual in each trial (De Cáceres et al. 2011). The niche centre was defined as the multivariate mean in the subspace of the two first axes, which together accounted for $>80 \%$ of the observed variation. To describe niche breadth while accounting for resemblance among resources, we used Rao's quadratic entropy as implemented in the 'indicspecies' package (De Cáceres \& Legendre 2009). In addition, we also estimated the proportion of diet variation of each population that was due to either variation within (WIC) or among individuals (TNW). To this purpose, we modified the $\mathrm{R}$ package "indicspecies" to be able to deal with the fact that some resources are more similar among them than others (De Cácereset al. 2011). Finally, we estimated the mean pairwise niche overlap between each pair of individuals as a measure of the overall similarity among individuals within a population (De Cáceres et al. 2011).

We used Bayesian general mixed-effects models to estimate the repeatability of food preferences, using the packages MCMCglmm (Hadfield 2009) and BRMS (Bürkner 2017). For both wild-trapped adults and captive-bred juveniles, we partitioned the within- vs. between-individual components of variance in food preferences by fitting individual identity as a random effect. The among-individual variance expressed as a proportion of the trait is the repeatability (Gamer et al., 2010). The confounding effect of sex, age, session, and aviary were evaluated by including them as fixed effects. Although individuals were tested in groups of six individuals, we did not include the testing group as random blocking effect because adding it did not improve model fit (Table S1). To assess whether morphology influenced food preferences of pigeons, we conducted a Principal Component Analysis (PCA) based on the correlation matrix of morphological traits with the function "prcomp" in R (R core team 2015), and used the scores as predictors in mixed-effects models.

We disentangled the genetic and environmental components underpinning variation in food preference using an animal model approach (Kruuk \& Hadfield 2007; Wilson et al. 2010), again based on Bayesian mixedeffects models. For labile traits, phenotypic variation at the between-individual level is not necessarily equal to additive genetic variation because individuals may also experience differing sets of non-genetic effects on their phenotypes. However, the between-individual phenotypic variation can be decomposed into genetic and non-genetic (or so-called 'permanent environmental') components using quantitative genetic models (Kruuk \& Hadfield 2007; Wilson et al. 2010). Thus, we used Bayesian Mixed Models that included individual identity plus two additional variables as random factors, one relating individuals to their records in the pedigree and another accounting for the nest where offspring were raised (as half offspring were cross-fostered). We estimated heritability by dividing the posterior distribution of the variance component associated with the pedigree by total variance.

We also used the "animal model" to analyse diet plasticity by means of reaction norms, comparing preferences during the short-term assays with those from the long-term assays.

Following Nussey et al. (2007), we assessed variation in plasticity of the niche centre among individuals by including the interaction between period (i.e. short-term vs long-term, coded as a fixed effect) and the identity 
of individuals (coded as a random effect). We compared this model with a model including the pedigree as a random effect to determine the existence of heritable variation in plasticity (i.e. adaptive plasticity). We compared models using Waic, the widely applicable information criterion (Bürkner 2017).

\section{Results}

Our food-choice experiment detected consistent variation among individuals in food preferences over a short period (Fig 1 ). The niche centre of each individual in the resource space - defined using the two first axes of a principal coordinate analysis (Fig. 1D ; Table S2 ) -was highly consistent over the trials (Table 1; Fig. 2 ) regardless of age, sex, trial order or aviary (Table S3 ). Despite striking differences in nutritional contents and size of the seeds used for this experiment (Fig 1B,C ), individuals only coincided in avoiding one food type (i.e. peas).

\section{Hosted file}

image1.emf available at https://authorea.com/users/377081/articles/493888-resourcepreferences-and-the-emergence-of-individual-niche-specialization-within-populations

Fig 1. Food characterization and preferences among wild pigeons. A. Density plots of food preferences for individual from the two studied populations. Food preferences are represented as the fraction of each food type consumed by an individual during a 20 -min trial $(N=158$ trials for 42 individuals). Trials for pigeons from Moià are shown in purple and those from BCN in green. B. Differences in nutritional and morphological similarity between food type (darker colors indicate larger differences). C. Characterization of nutritional and morphological differences between food types.D. Niche position and breadth of the two studied wild populations (mean and standard error) in the space defined by resource differences.

Table 1. Repeatability of the niche centre and breadth in the short and long-term . Repeatability is the intra-class coefficient of each measure (i.e. phenotypic variation among individuals/total phenotypic variation). The abbreviations l-95\% CI and u-95\% CI refer to the lower and upper 95\% credibility interval, respectively. The long-term assay was conducted one year after the short-term assay.

\begin{tabular}{llllllll}
\hline & PCoA 1 & PCoA 1 & PCoA 1 & PCoA 2 & PCoA 2 & PCoA 2 & PCoA 2 \\
\hline Short-term assays & Repeatability & $\mathbf{l - 9 5 \%} \mathbf{C I}$ & $\mathbf{u - 9 5 \%} \mathbf{C I}$ & Repeatability & $\mathbf{l - 9 5 \%}$ CI & u-95\% CI \\
MOIA & & & & & & \\
BCN & 0.85 & 0.74 & 0.93 & 0.87 & 0.77 & 0.94 \\
Captivity & 0.72 & 0.56 & 0.85 & 0.85 & 0.74 & 0.93 \\
Long-term assays & 0.87 & 0.79 & 0.92 & 0.87 & 0.8 & 0.93 \\
MOIA & 0.82 & 0.25 & 0.97 & & & \\
BCN & 0.97 & 0.89 & 0.99 & 0.57 & 0.01 & 0.92 \\
Captivity & 053 & 0.26 & 0.74 & 0.94 & 0.81 & 0.99 \\
\hline
\end{tabular}

\section{Hosted file}

image2.emf available at https://authorea.com/users/377081/articles/493888-resourcepreferences-and-the-emergence-of-individual-niche-specialization-within-populations

Fig 2. Variation among individuals in food preferences, considering energetic and nutritional implications. The plots show individual-level random effects of Bayesian Mixed Models, estimated using four replicates per individual. The red line represents the average value of the population. The multidimensional niche centre is represented by PCoA 1 and PCoA 2.

If energy value were the only driver of preferences, we would expect that pigeons mostly consumed sunflower seeds, which are richer in fat than any other tested food (i.e. they should show higher values of PCoA 1; see Table S2 ). However, only some individuals showed strong preferences for sunflower; others instead 
preferred cereal, notably wheat (Figs. 1A, 2, S1 ). The energy density of cereal is lower, due to the reduced fat contents, but they are richer in carbohydrates. The breakdown and absorption of carbohydrates is much faster than fat, providing energy that can rapidly be used by the animal. A bi-variate Bayesian Mixed Model revealed that preferences for carbohydrates and fat were negatively correlated among individuals, a correlation that was independent of the amount of food consumed during the trial (MOIA: Correlation $=-0.95,95 \% \mathrm{CI}=-0.98$ to -0.88 ; BCN: Correlation $=-0.99,95 \% \mathrm{CI}=-0.98$ to -0.99$)$. While energy has traditionally been considered the primary currency influencing food choice, our results suggest that the relative amounts of various nutrients is also relevant (Fig. 2 ).

Besides nutrient contents, food choice also appeared to be influenced by morphological characteristics of the food. Of the three cereal grains, wheat was generally preferred over popcorn and oats. The main difference among these food types was their size. Pigeons use both size and number of items in assessing the amount of food, but they weight number more heavily than size (Shettleworth 1987b). It follows that when provided in the same amount, they should prefer the smaller seed -i.e. wheat. Again, however, some individuals consistently preferred corn over wheat during the four trials, reinforcing the view that individuals remain selective even when the searching and handling costs of using alternative resources is negligible.

Having established consistent individual differences in food preferences, we estimated the contribution of individual niche breadth (WIC) to the total niche breadth of the population (TNW). We used Rao's quadratic entropy to estimate WIC and TNW while considering nutritional and morphological differences between the resources. We found that the niches of individuals represented, on average, $59 \%$ of the niche breadth of the population (mean $\pm \mathrm{WIC} / \mathrm{TNW}=0.68 \pm 0.51$ for MOIA and $0.51 \pm 0.35$ for BCN). Thus, individual niche widths were considerably narrower than the niches of their populations, a pattern that was consistent despite striking ecological differences among populations (Sol 2008). However, we also detected substantial niche overlap between each individual and the remaining individuals of the population $(75.4 \% \pm 24.7$ for BCN and $88.9 \pm 15.8$ for MOIA). This overlap indicates that within a population the majority of individuals shared similar food preferences (Fig. 2 ). It also reflects that the studied populations included both specialists and generalists (Table 1, Fig S2).

Variation among individuals in food preferences may be ephemeral if they reflect differences in the physiological condition of the individual, for example regarding its nutritional state. Indeed, the amount of food consumed was highly repeatable across individuals (Fig. 2 ). This suggests that some individuals were more motivated than others to eat. However, we found no evidence that the niche centre of individuals was influenced by their body condition (Table S4 ) or by the total amount of food consumed during an assay (effect size $>0.01[ \pm 0.1]$ in all cases). We neither found that fasting makes individuals either more discriminant or more willing to accept a wider variety of foods (Moon \& Zeigler 1979). Indeed, choices in the first trial of the day, when the individual had not consumed any food in the previous $12 \mathrm{~h}$, were highly consistent with choices in the second trial - when individuals have had that chance to already eat for 20 minutes (Table S3 ). These findings do not necessarily contradict the view that hungry animals make sub-optimal food choices (Moon \& Zeigler 1979), as before the experiments food was providedad libitum. Therefore, it was unlikely that any individual was in bad body condition.

Long-term consistencies in food preferences among individuals are expected if these are either heritable or learned individually or in a social context. To disentangle genetic and environmental effects, we examined the preferences of birds raised in captivity. Like in the case of wild pigeons, captive-bred individuals also exhibited consistent variation in food preferences despite being raised under similar environmental conditions (Fig. 2, Table 1 ). However, the cross-fostering experiment revealed that the additive genetic variance component of the niche centre was low $\left(\mathrm{PCoA} 1: h^{2}=0.082, \mathrm{CI}_{95 \%}=0.000\right.$ to $0.375 ; \mathrm{PCoA} 2: h^{2}=0.041, \mathrm{CI}_{95 \%}=$ 0.011 to 0.256$)$. The niche centre was indeed little affected by more stable state variables like body size and beak shape (Tables S6, S7 ), which have high genetic influences $\left(h^{2}>0.7\right.$, Authors unpublished). Likewise, we found that the effect of the common rearing environment was also low $\left(\mathrm{PCoA} 1: e^{2}=0.001, \mathrm{CI}_{95 \%}=\right.$ 0.021 to $0.214 ; \mathrm{PCoA} 2: e^{2}=0.047, \mathrm{CI}_{95 \%}=0.015$ to 0.153 ), suggesting that diet was little influenced by the vertical transmission of information from the parents. Together, additive genetic and common environmental 
variances accounted for $>14 \%$ of all phenotypic variance, and hence only marginally contributed to variation in niche centre (Table S5 ).

Not surprisingly, the food preferences of individuals were not conserved after one year (Fig 3 ). During the long-term assays individuals still showed consistency in their food preferences across trials (Table 1 ), yet these preferences were different from those observed during the short-term assays (Figs. 3, $\mathbf{4}$ ). These changes in preferences did not reflect heritable variation in diet plasticity (Table S8 ). However, a role for learning is suggested in the tendency of individuals to converge over time toward a diet richer in fat (i.e. more caloric) and proteins (Fig. 4 ). If niche shifts were driven by random processes, we would not expect such a convergence. Thus, the extent to which the niche centre changed over time did not seem to reflect variation in responsiveness (i.e. ability to accommodate the behaviour to current conditions). Rather, it appears to be mainly determined by the distance to the "optimum" to which individuals tended to converge, changing on average more for wild pigeons than for those raised in captivity.

\section{Hosted file}

image3.emf available at https://authorea.com/users/377081/articles/493888-resourcepreferences-and-the-emergence-of-individual-niche-specialization-within-populations

Fig. 3. Individual differences in niche centre between the short- and long-term assays of food preferences. Correlations have been estimated with a bi-variate Bayesian Mixed Models including period (short-term vs long-term) as fixed effect and individual as random effect. Correlations are the mode and HPD intervals (within brackets) estimated by means of a bi-response Bayesian mixed model of PCoAs in the short- and long-term assays. Dots with no line indicate individuals for which no long-term assay was conducted.

\section{Hosted file}

image4.emf available at https://authorea.com/users/377081/articles/493888-resourcepreferences-and-the-emergence-of-individual-niche-specialization-within-populations

Fig. 4. Changes in the niche centre, amount of food consumed and food properties between the short-term and long-term assays of food preferences. The plots show the estimates and standard error of the predicted responses of the model. The amount of total food, calories and nutrients consumed is in grams. Results for PCoA 2 are shown in Fig. S3.

Unlike niche centre, niche breadth only changed between the short and long-term assays in pigeons from MOIA (Fig. 5 ). In the short-term assays, their WIC was smaller than that of individuals from BCN and those raised in captivity. One year after, however, WIC had expanded to levels similar to those of the other two groups. Although individual niche expansion was also accompanied by an increase in population niche breadth (WIC), the ratio WIC/TNW increased around $48 \%$ from the short- to the long-term assays. This means that individuals used a larger fraction of the resources exploited by the entire population than they did one year before. While individuals from BCN and those raised in captivity did not show similar niche expansions, the ratio WIC/TNW also increased substantially as a result of a decrease in the population niche breadth. Thus, the degree of resource specialization within the population diminished over time.

\section{Hosted file}

image5.emf available at https://authorea.com/users/377081/articles/493888-resourcepreferences-and-the-emergence-of-individual-niche-specialization-within-populations

Fig. 5. Changes in individual (WIC) and total population (TNW) niche breadth, between the short-term and long-term assays of food preferences. The plot on the left shows the estimates and standard error of the predicted responses. The plot on the right describes changes in mean WIC and TNW. Arrows have been added to help identify the direction of the change (i.e. from the values of short-term assays to those of the long-term assays). 


\section{Discussion}

Our work adds new evidence to the increasing bulk of papers reporting that individuals sharing a common environment can exhibit consistent differences in resource-utilization niche (Bolnick et al. 2003), challenging the traditional idea that individuals within a population are all ecologically equivalent (Colwell et al. 1971). More importantly, while the vast majority of cases of individual specialization document realized individual specialization (sensu Bolnick et al. , 2003a), our experimental demonstration that individuals exposed to similar conditions may differ in food preferences provides a more mechanistic understanding for the existence of niche variation among individuals.

The existence of individual specialization in feral pigeons was first suggested by Giraldeau and Lefebvre (1985). These authors analysed the crop contents of individuals baited with a mixture of seeds, and noticed a strong degree of individual variation in the seeds consumed. Our common garden experiments using individuals from two ecologically distinct populations not only support their finding, but also suggest that specializations reflect consistent differences among individuals in food preferences. Our analyses further reveal that individual specialization was not associated with age, sex or morphological traits like body size and beak morphology. Instead, individuals seemed to use different optimization criteria in their choice of foods, some favouring compounds that provide fast energy while others prefer compounds with higher energy but more difficult to break down.

Resource specialization was only consistent over short time spans (days) but did not last for longer periods (one year). Short-term consistencies are expected if preferences are driven by the physiological state of individuals. Although individuals used different optimization criteria in resource-choice, we did not find evidence that individuals in worse body condition consumed larger amounts of food or foods that provided more rapid energy. Animals may improve foraging efficiency by developing searching images for a few food types (Pietrewicz \& Kamil 1979), yet this also does not seem a likely explanation for specialization because in our assays food types were provided in separated patches.

Given the low stability of food preferences, it is unsurprising that we found little evidence for additive genetic effects. Our estimations of the heritable component of food preferences were always low, even when they may include maternal effects. Our analyses neither indicated that preferences were linked to heritable state variables, such as body size and beak morphology. The alternative that preferences were learned from their parents also seem unlikely. First, individuals born in captivity were raised under similar conditions, and hence their early food experiences were similar. Second, the cross-fostering experiment showed a low effect of the common rearing environment on food preferences, suggesting that diet was little influenced by vertical transmission of information from the parents.

The fact that food preferences were little constrained by genetic architecture and/or learning was reflected in their plastic nature. Preferences of individuals did not only change after one year in captivity, but also tended to converge toward similar resources. These adjustments did not seem to reflect adaptive plasticity, as suggests the low heritability of individuals' responsiveness between the short and long-term assays. However, a role for learning is suggested in the tendency of individuals to converge over time toward a diet richer in calories and proteins. If niche shifts were driven by random processes, we would not expect this convergence.

Regardless of the cause, the finding that food preferences are highly plastic is important because it challenges a major mechanism that may generate and maintain niche specialization within a population. In our study, plastic adjustments of food preferences led to a substantial decrease in the degree of food specialization within the population, either as a result of an expansion of the niche of individuals or a reduction of population niche breadth. Still, the finding that variation in food preferences may easily emerge among individuals subject to similar conditions is relevant because initial decisions regarding what to eat may largely shape the future diet in the wild. Early preferences in the use of certain foods can give rise to different experiences that may reinforce (if positive) the initial preferences (Tinker et al. 2009). Given that foraging proficiency often increases with experience (i.e., learning), initial differences in food preferences among individuals may limit the use of alternative foods that require to learn new foraging skills (Partridge \& Green 1987). Variation 
in resource preferences may also be maintained by emotional responses, like the aversion to explore and incorporate novel foods once the individual reaches maturity (Greenberg \& Mettke-hofmann 2001).

We suggest that the above effects may not have been detected in our experiments for two main reasons. First, our food-choice assays were designed to measure preferences, and hence were little demanding in terms of other foraging components like searching, identifying and handling foods. This may have reduced the costs of shifting among food resources. Second, the exposure of individuals to the same stimuli for long periods may have favoured the convergence toward similar food preferences in captivity. Experiments in rodents have for instance shown that individuals raised under stable food conditions are more selective in food choice than those raised under fluctuating food conditions (Gray 1981).

Simultaneous choices between different types of exactly the same amount of food must hardly occur in nature. Rather, resource supplies are likely to vary in time and space, exposing individuals to different experiences and uncertainties regarding food. Moreover, the need of searching, identifying, and handling foods makes it unlikely that individuals are able to exploit all food types efficiently (Price 1987), particularly when this requires advanced cognition (Tinker et al.2009). The costs of acquiring resource information may also force individuals to make decisions based on the perceived rather than actual perceptions of risk (Blumstein 2006; Lamanna \& Martin 2016). Under these circumstances, the retention of initial resource preferences through learning trade-offs and neophobic responses is more plausible. In sea otters (Enhydra lutris ), resource specialization driven by reduced food availability is not associated with morphological or genetic differences between individuals, but it appears to reflect limitations in their capacity to learn the skills needed to efficiently exploit different preys (Tinker et al. 2009).

Although both laboratory and field studies may provide important insight into the origin and maintenance of resource specialization, each of these approaches is limited in scope. Resource preferences are easier to study in common garden experiments. However, the conditions individuals find in captivity may largely differ from those they encounter in nature. Thus, the integration of laboratory and field studies may largely broaden our understanding of the role of resource preferences in shaping resource specialization within animal populations.

Acknowledgments. We thank Louis Lefebvre for past discussions about pigeon's behaviour, and Domingo Rodriguez Teijeiro and the staff of Pedro Pons for allowing us to conduct the experiments within their facilities. This project was funded by grants from the Spanish Government CGL2013-47448-P and CGL201790033-P. OL and CGL were supported, respectively, by FPI (BES2008-007095) and AGAUR (FI-DGR 2009) grants.

Araújo, M.S., Bolnick, D.I. \& Layman, C.A. (2011). The ecological causes of individual specialisation. Ecol Lett , 14, 948-958.

Biedermann, T., Garlick, D. \& Blaisdell, A.P. (2012). Food choice in the laboratory pigeon. Behav Processes , 91, 129-132.

Blumstein, D.T. (2006). Developing an evolutionary ecology of fear: How life history and natural history traits affect disturbance tolerance in birds. Anim Behav , 71, 389-399.

Bolnick, D.I., Amarasekare, P., Araújo, M.S., Reinhard Bürger, Levine, J.M., Novak, M., et al. (2011). Why intraspecific trait variation matters in community ecology. Trends Ecol Evol , 26, 183-192.

Bolnick, D.I., Svanback, R., Fordyce, J. a, Yang, L.H., Davis, J.M., Hulsey, C.D., et al. (2003). The ecology of individuals: incidence and implications of individual specialization. Am Nat, 161, 1-28.

Burkner, P.-C. (2017). brms : An R Package for Bayesian Multilevel Models Using Stan. J Stat Softw , 80, $1-28$.

De Caceres, M. \& Legendre, P. (2009). Associations between species and groups of sites: indices and statistical inference. Ecology, 90, 3566-3574. 
De Caceres, M., Sol, D., Lapiedra, O. \& Legendre, P. (2011). A framework for estimating niche metrics using the resemblance between qualitative resources. Oikos , 120, 1341-1350.

Colwell, R.K., D. \& Futuyma. (1971). On the measurement of niche breadth and overlap. Ecology , 52, $567-76$.

Dall, S.R.X., Bell, A.M., Bolnick, D.I. \& Ratnieks, F.L.W. (2012). An evolutionary ecology of individual differences. Ecol Lett , 15, 1189-1198.

Dingemanse, N.J. \& Wolf, M. (2010). Recent models for adaptive personality differences: a review. Philos Trans R Soc Lond B Biol Sci, 365, 3947-58.

Dridi, S. \& Lehmann, L. (2015). A model for the evolution of reinforcement learning in fluctuating games.

Giraldeau. (1984). Group foraging: The skill pool effect and frequency dependent effect. Am Soc Nat .

Giraldeau, L.-A. \& Lefebvre, L. (1985). Individual feeding preferences in feral groups of rock doves. Can $J$ Zool , 63, 189-191.

Gray, L. (1981). Genetic and experiential differences affecting foraging behavior. In: Foraging behavior: ecological, ethological and psychological approaches (eds. Kamil, A. \& Sargent, T.). Garland, NY, pp. $455-473$.

Greenberg, R. \& Mettke-hofmann, C. (2001). Ecological Aspects of Neophobia and neophilia in birds . Curr Ornithol Vol 16 .

Hadfield, J.D. (2009). MCMCglmm: Markov chain Monte Carlo methods for Generalised Linear Mixed Models, 1-25.

Houston, A. \& McNamara, J. (1999). Models of adaptive behaviour: an approach based on state . Cambridge University Press, Cambridge, UK.

Inman, A.J., Lefebvre, L. \& Giraldeau, L.-A. (1987). Individual diet differences in feral pigeons: Evidence for resource partitioning. Anim Behav , 35, 1902-1903.

Johnson, D.H. (1980). The Comparison of Usage and Availability Measurements for Evaluating Resource Preference. Ecology .

Johnston, R. \& Janiga, M. (1995). Feral pigeons . Oxford Univesrity Press, New York, USA.

Kamil, A.C. (1983). Optimal foraging theory and the psychology of learning. Integr Comp Biol .

Kruuk, L.E.B. \& Hadfield, J.D. (2007). How to separate genetic and environmental causes of similarity between relatives. J Evol Biol, 20, 1890-1903.

Lamanna, J.A. \& Martin, T.E. (2016). Costs of fear: Behavioural and life-history responses to risk and their demographic consequences vary across species. Ecol Lett , 19, 403-413.

Luttbeg, B. \& Sih, A. (2010). Risk, resources and state-dependent adaptive behavioural syndromes. Philos Trans R Soc B Biol Sci, 365, 3977-3990.

Machovsky-Capuska, G.E., Miller, M.G.R., Silva, F.R.O., Amiot, C., Stockin, K.A., Senior, A.M., et al. (2018). The nutritional nexus: Linking niche, habitat variability and prey composition in a generalist marine predator. J Anim Ecol , 87, 1286-1298.

Moon, R.D. \& Zeigler, H.P. (1979). Food preferences in the pigeon (Columba livia). Physiol Behav , 22, $1171-1182$.

Nussey, D.H., Wilson, A.J. \& Brommer, J.E. (2007). The evolutionary ecology of individual phenotypic plasticity in wild populations. J Evol Biol , 20, 831-844. 
Partridge, L. \& Green, P. (1987). An advantage for specialist feeding in jackdaws, Corvus monedula. Anim Behav , 35, 982-990.

Pietrewicz, A.T. \& Kamil, A.C. (1979). Search image formation in the blue jay (Cyanocitta cristata). Science (80-).

Price, T. (1987). Diet Variation in a Population of Darwin's Finches.Ecology , 68, 1015-1028.

Pyke, G.H. (1984). Optimal foraging theory: a critical review.Annu Rev Ecol Syst Vol 15 , 523-575.

Schoener, T.W. (1971). Theory of Feeding Strategies. Annu Rev Ecol Syst , 2, 369-404.

Schoener, T.W. (1986). Resource Partitioning. In: Community Ecology: Pattern and Process (ed. Kikkawa, J. \& Anderson, D.J.). Blackwell Scientific, Boston, MA, pp. 91- 126.

Shettleworth, S.J. (1985). Handling Time and Choice in Pigeons. J Exp Anal Behav , 44, 139-155.

Shettleworth, S.J. (1987a). Individual differences in choice of food items by pigeons. Behav Processes, 14, 305-318.

Shettleworth, S.J. (1987b). Learning and foraging in pigeons: Effects of handling time and changing food availability on patch choice. In:Quantitative analyses of behavior, Vol. 6. Foraging (eds. Commons, M.L., Kacelnik, A. \& Shettleworth, S.J.). Lawrence Erlbaum Associates, Inc., pp. 115-132.

Slagsvold, T. \& Wiebe, K.L. (2007). Learning the ecological niche.Proc R Soc B Biol Sci , 274, 19-23.

Sol, D. (2008). Artificial selection, naturalization, and fitness: Darwin's pigeons revisited. Biol J Linn Soc , 93, 657-665.

Sol, D., Elie, M., Marcoux, M., Chrostovsky, E., Porcher, C. \& Lefebvre, L. (2005). Ecological mechanisms of a resource polymorphism in Zenaida Doves of Barbados. Ecology , 86, 2397-2407.

Tinker, M.T., Bentall, G. \& Estes, J.A. (2008). Food limitation leads to behavioral diversification and dietary specialization in sea otters.Proc Natl Acad Sci, 105, 560-565.

Tinker, M.T., Mangel, M. \& Estes, J.A. (2009). Learning to be different: Acquired skills, social learning, frequency dependence, and environmental variation can cause behaviourally mediated foraging specializations. Evol Ecol Res , 11, 841-869.

Van Valen, L. (1965). Morphological Variation and Width of Ecological Niche. Am Nat, 99, 377-390.

Wilson, A.J., Reale, D., Clements, M.N., Morrissey, M.M., Postma, E., Walling, C.A., et al. (2010). An ecologist's guide to the animal model. J Anim Ecol , 79, 13-26.

Woo, K.J., Elliott, K.H., Davidson, M., Gaston, A.J. \& Davoren, G.K. (2008). Individual specialization in diet by a generalist marine predator reflects specialization in foraging behaviour. J Anim Ecol, 77, 1082-1091.

\section{SUPPORTING INFORMATION}

Additional supporting information may be found online in the Supporting Information section. 